SALES JÚNIOR, R.; ITO, S.C.S.; ROCHA, J.M.M.; SALVIANO, A.M.; AMARO FILHO, J.; NUNES, G.H.S. Aspectos quantitativos e qualitativos de melão cultivado sob doses de fertilizantes orgânicos. Horticultura Brasileira, Brasília, v.23, n.3, p.718-721, jul-set 2005.

\title{
Aspectos quantitativos e qualitativos de melão cultivado sob doses de fertilizantes orgânicos
}

\author{
Rui Sales Júnior ${ }^{1}$; Silvio C.S. Ito ${ }^{1}$; Joyce Michelle de M. Rocha ${ }^{1}$; Alessandra M. Salviano ${ }^{2}$; Joaquim \\ Amaro Filho'; ${ }^{2}$ Glauber Henrique S. Nunes ${ }^{1}$ \\ ESAM, Depto. de Ciências Vegetais'; Ciências Ambientais², C. Postal 137, 59625-900 Mossoró-RN; E-mail: ruisales@esam.br
}

\begin{abstract}
RESUMO
A produtividade e qualidade do melão cultivado sob doses de fertilizantes orgânicos foram avaliados em experimento realizado de agosto a novembro de 2002 em campos de produção comercial no estado do Ceará. O híbrido de melão AF 646 (sementes Sakata) foi semeado diretamente com espaçamento de dois metros entre linhas e meio metro entre plantas. $\mathrm{O}$ delineamento experimental foi em blocos ao acaso com quatro repetições. Os tratamentos avaliados foram: 1) $100 \mathrm{~g}$ de Ribumin ${ }^{\circledR}$ por metro linear; 2) $150 \mathrm{~g} \mathrm{de}$ Ribumin ${ }^{\oplus}$ por metro linear 3) $200 \mathrm{~g}$ de Ribumin ${ }^{\circledR}$ por metro linear; 4) $1400 \mathrm{~g}$ de composto Pole ${ }^{\circledR}$ por metro linear; 5) $700 \mathrm{~g}$ de composto Pole $^{\circledR}+100$ g Ribumin ${ }^{\circledast}$ por metro linear, respectivamente e 6) testemunha sem adição de composto. Todos os tratamentos foram aplicados um dia antes da semeadura. Após a colheita os frutos foram contados e uma amostra aleatória de quatro frutos por parcela foi retirada para análise no laboratório. As variáveis analisadas foram peso dos frutos (PF), peso estimado da produtividade (PE) diâmetros longitudinal (DL) e transversal dos frutos (DT), espessura de polpa (EP), teor de sólidos solúveis totais (SST) e firmeza de polpa (FP). A produtividade foi calculada com os dados de número de frutos (NF) por parcela e peso médio de frutos. Avaliou-se também o custo de produção. A análise de variância revelou significância para as variáveis PF, PE, DL, EP e SST. As médias do DL e da EP e SST apresentaram diferença significativa entre a testemunha e os demais tratamentos que não diferiram entre si. Apesar de não ter sido constatada diferença estatística significativa entre as médias de produtividade, para os tratamentos onde se adicionou fertilizante orgânico, observou-se que os tratamentos nos quais foram utilizados o fertilizante Ribumin ${ }^{\oplus}$ apresentaram os menores custos de produção.
\end{abstract}

Palavras-chave: Cucumis melo, sólidos solúveis totais, firmeza de polpa, fertilizantes orgânicos, análise de custo

\begin{abstract}
Quantitative and qualitative aspects of the use of organic fertilizers on cultivated melon

The yield and quality of melon fruits cultivated under different doses of organic fertilizers were evaluated. The field trial was conducted from August to November 2002, in Ceará State, Brazil. The experimental design was made of random blocks of four replications. Melon hybrid AF 646 (Sakata Seeds) was sown using a distance between-beds of two meters and $0,5 \mathrm{~m}$ between plants. Six treatments were evaluated 1) $100 \mathrm{~g}$ of Ribumin ${ }^{\circledR}$ in a linear meter; 2) $150 \mathrm{~g}$ of Ribumin ${ }^{\circledR}$ in a linear meter; 3) $200 \mathrm{~g}$ of Ribumin ${ }^{\circledR}$ per linear meter; 4) $1400 \mathrm{~g}$ of Pole ${ }^{\circledR}$ per linear meter; 5) $700 \mathrm{~g}$ of Pole ${ }^{\circledR}+100$ $\mathrm{g}$ of Ribumin ${ }^{\oplus}$ in a linear meter and 6) Untreated control. All the treatments were applied one day before seeding. After harvest, fruits were counted and a random sample of four fruits per plot was taken for analysis into the laboratory. The analyzed variables were: fruit weight (PF), longitudinal diameter (DL) and traverse diameter (DT), pulp thickness (EP), total soluble solids content (SST) and pulp firmness (FP). Yield was calculated from data of fruit number per plot and their mean weight. The variance analysis revealed significant difference for PF, PE, DL, EP and SST. The mean values for DL, EP and SST parameters of the treated group were significantly different from the mean of the untreated control. No significant difference was observed between samples from the treated group. Nevertheless Ribumin ${ }^{\circledR}$ fertilizer provided smaller production costs.
\end{abstract}

Keywords: Cucumis melo, total soluble solids, pulp firmness, organic fertilizers, cost analysis.

\section{(Recebido para publicação em 1 de abril de 2004 e aceito em 9 de maio de 2005)}

$\mathrm{O}$ melão (Cucumis melo L.), adapta-se bem a regiões caracterizadas por climas quentes e com alta intensidade luminosa. Os estados do Rio Grande do Norte e Ceará apresentam excelentes condições edafoclimáticas para o seu cultivo, o que possibilitou no ano de 2003, exportar aproximadamente 150 mil toneladas de frutos, gerando receita, ao redor dos 58 milhões de dólares (SECEX/DTIC, 2004). Diante da importância desta cultura para a região, há uma grande demanda de informações visando definir um sistema produtivo que apresente redução de custos, aumente a produtividade, e alcance os padrões mínimos de qualidade dos frutos exigidos no mercado internacional, sendo este um dos grandes desafios da fruticultura brasileira.

Em melão, o termo qualidade tem sido relacionado a diversos fatores. Uma das características mais estudadas é o teor de sólidos solúveis totais (SST), fator tradicionalmente utilizado para assegurar sua qualidade (PROTADE, 1995). A firmeza da polpa é outra variável importante que indica a sua resis- tência ao transporte e a vida de prateleira, estando relacionado com o "flavor", que é perceptível pelo paladar (MENEZES et al., 1998).

É notório que, para que se produzam frutos com qualidade, capazes de competir em um mercado de economia globalizada, é de suma importância a utilização de insumos energéticos externos, caracterizados pela aplicação intensiva de fertilizantes no solo. Nos plantios comerciais, as adubações minerais e orgânicas são usadas em larga escala havendo, portanto, a necessidade de se 
definir os melhores produtos e doses a serem aplicados, no intuito de maximizar os lucros, a produtividade e a qualidade final dos frutos.

A matéria orgânica, pelo seu efeito na melhoria das condições químicas, físicas e biológicas do solo, contribui para o aumento da produtividade e para a melhor qualidade dos frutos. Em melão tipo Amarelo, a utilização de composto orgânico, principalmente em solos arenosos, pode aumentar a produtividade, a quantidade de frutos de primeira qualidade e o teor de sólidos solúveis (MENEZES et al., 2000). No entanto, quando se utiliza grande quantidade de matéria orgânica, as condições para o surgimento de doenças são favorecidas, em função do aumento da capacidade de retenção de água do solo. Além disso, Fabeiro et al. (2002) encontraram uma relação inversa entre quantidade de água aplicada e a produção e qualidade de melão em região semi-árida.

Frente ao exposto, este trabalho teve como objetivo avaliar a produção e a qualidade do híbrido de melão AF 646 da empresa Sakata, cultivado sob diferentes doses de fertilizantes orgânicos.

\section{MATERIAL E MÉTODOS}

O experimento foi realizado de agosto a novembro de 2002 em um Neossolo Quartzarênico em campos de produção comercial de melão em Icapuí (CE). Amostras coletadas na profundidade de 0-20 cm foram submetidas à análise química e física para sua caracterização. $\mathrm{O}$ pH foi determinado em água na relação 1:2,5 de solo:solução. O potássio e o sódio trocáveis foram determinados por fotometria de chama, após extração com solução de $\mathrm{HCl} 0,05 \mathrm{~mol} / \mathrm{L}$ + $\mathrm{H}_{2} \mathrm{SO}_{4}$ 0,025 mol/L (Mehlich-1), enquanto o cálcio, magnésio e alumínio trocáveis foram extraídos com $\mathrm{KCl}$ $1 \mathrm{~mol} / \mathrm{L}$ e determinados por titrimetria. O fósforo foi determinado por colorimetria usando ácido ascórbico como redutor, após a extração com Mehlich-1. Todos conforme EMBRAPA (1997). As análises apresentaram os seguintes resultados: $\mathrm{pH}=$ 6,$0 ; \mathrm{P}=15 \mathrm{mg} / \mathrm{dm}^{3} ; \mathrm{K}^{+}=0,13 \mathrm{cmol}_{\mathrm{c}} / \mathrm{dm}^{3}$; $\mathrm{Ca}^{2+}+\mathrm{Mg}^{2+}=0,90 \mathrm{cmol}_{\mathrm{c}} / \mathrm{dm}^{3} ; \mathrm{Al}^{3+}=$ $0,05 \mathrm{cmol} / \mathrm{dm}^{3} ; \mathrm{Na}^{+}=0,20 \mathrm{cmol} / \mathrm{dm}^{3}$.
Na caracterização física foram determinados a granulometria, com o uso do dispersante hexametafosfato de sódio, pelo método da pipeta e a densidade do solo pelo método do anel volumétrico segundo metodologia da Embrapa (1997); a retenção de água foi determinada pelo método da placa e membrana de pressão (RICHARDS, 1949), apresentando os seguintes resultados: areia grossa $=68 \mathrm{dag} / \mathrm{kg}$; areia fina $=24,7 \mathrm{dag} / \mathrm{kg} ;$ silte $=2,4 \mathrm{dag} / \mathrm{kg}$; argila $=4,8 \mathrm{dag} / \mathrm{kg} ; \mathrm{d}=1,34 \mathrm{~kg} / \mathrm{dm}^{3 ;}$ água retida a $0,01 \mathrm{MPa}=5,06 \mathrm{~g} / \mathrm{kg}$; água retida a $15 \mathrm{Mpa}=1,57 \mathrm{~g} / \mathrm{kg}$.

A cultivar de melão utilizada foi o híbrido AF 646 (sementes Sakata), atualmente um dos mais utilizados na região. Todos os tratamentos receberam uma adubação uniforme aplicando-se, em fundação, $80 \mathrm{~kg} /$ ha de $\mathrm{P}_{2} \mathrm{O}_{5}$ como superfosfato simples. O N (90 kg/ha) e o K (120 kg/ha de $\left.\mathrm{K}_{2} \mathrm{O}\right)$ foram parcelados em quantidades iguais para serem aplicados via água de irrigação, utilizando-se como fontes o cloreto de potássio e a uréia, respectivamente. A freqüência de aplicação foi a cada 4 dias durante o período de 3 a 45 dias após a emergência para K, e de 3 a 55 dias para N.

O plantio foi efetuado via semeadura direta, sendo adotada a distribuição de 2:1, ou seja, em um dos gotejadores havia duas plantas e no outro uma, e assim sucessivamente, totalizando 3 plantas por metro linear. O espaçamento utilizado foi de dois metros entre linhas e meio metro entre plantas. Os gotejadores apresentavam vazão de 1,5 $\mathrm{L} \mathrm{h}^{-1}$ e pressão de $10 \mathrm{~m}$ de coluna de água. As irrigações foram feitas diariamente, com base na evaporação de água do tanque classe $\mathrm{A}$ e no coeficiente de cultivo da cultura $(\mathrm{Kc})$ determinados por Miranda e Bleicher (2001). Os tratamentos foram aplicados no sulco de plantio de aproximadamente 3,0 cm de profundidade ao lado da mangueira de irrigação, um dia antes da semeadura. Os tratamentos constaram de: três doses crescentes de Ribumin ${ }^{\circledR}$ (para os tratamentos 1 a 3, respectivamente, 100; $150 \mathrm{e}$ $200 \mathrm{~g}$ por metro linear); 4) $1400 \mathrm{~g}$ de composto Pole ${ }^{\circledR}$ por metro linear; 5) 700 g de composto Pole ${ }^{\circledR}+100$ g Ribumin ${ }^{\circledR}$ por metro linear, e 6) testemunha absoluta sem adubação orgânica (Ribumin ${ }^{\circledR}$ ou composto Pole ${ }^{\circledR}$ ). As doses dos adubos seguiram a recomendação dos fabricantes. Os adubos orgânicos utilizados foram submetidos à análise química, onde para Pole ${ }^{\circledR}$ se obteve: $\mathrm{pH}=13,4$; $\mathrm{P}=11596 \mathrm{mg} / \mathrm{dm}^{3} ; \mathrm{K}^{+}=193,34 \mathrm{cmol}_{\mathrm{c}} /$ $\mathrm{dm}^{3} ; \mathrm{Ca}^{2+}=70,0+\mathrm{Mg}^{2+}=0,0 \mathrm{cmol} / \mathrm{dm}^{3}$; $\mathrm{Al}^{3+}=0,0 \mathrm{cmol} / \mathrm{dm}^{3} ; \mathrm{Na}^{+}=28,8 \mathrm{cmol} /$ $\mathrm{dm}^{3} ; \mathrm{CE}=132 \mathrm{dS} / \mathrm{m} ; \mathrm{C}=30,3 \mathrm{~g} / \mathrm{kg}$; $\mathrm{MO}=52,30 \mathrm{~g} / \mathrm{kg} ; \mathrm{N}=28,8 \mathrm{~g} / \mathrm{kg} ; \mathrm{C} /$ $\mathrm{N}=1,05$ e, para Rubumin ${ }^{\circledR}$ se obteve: $\mathrm{pH}=4,8 ; \mathrm{P}=22 \mathrm{mg} / \mathrm{dm}^{3} ; \mathrm{K}^{+}=1,81 \mathrm{cmol}_{\mathrm{c}} /$ $\mathrm{dm}^{3} ; \mathrm{Ca}^{2+}=27,50+\mathrm{Mg}^{2+}=0,50 \mathrm{cmol}_{\mathrm{c}} /$ $\mathrm{dm}^{3} ; \mathrm{Al}^{3+}=0,10 \mathrm{cmol} / \mathrm{dm}^{3} ; \mathrm{Na}^{+}=2,82$ $\mathrm{cmol} / \mathrm{dm}^{3} ; \mathrm{CE}=3,78 \mathrm{dS} / \mathrm{m} ; \mathrm{C}=12,46 \mathrm{~g} /$ $\mathrm{kg} ; \mathrm{MO}=21,48 \mathrm{~g} / \mathrm{kg} ; \mathrm{N}=0,15 \mathrm{~g} / \mathrm{kg} ; \mathrm{C} /$ $\mathrm{N}=83,10$. O carbono orgânico (C) foi determinado por oxidação a quente com dicromato de potássio e titulação com sulfato ferroso amoniacal, segundo método modificado de Walkley e Black (1934). O N total foi determinado pela digestão do fertilizante com ácido sulfúrico e água oxigenada, seguida de destilação a vapor (Kjeldhal) com hidróxido de sódio e titulação usando como indicador o ácido bórico e ácido clorídrico. A condutividade elétrica foi determinada na relação 1:5 de fertilizante:água destilada. As demais análises seguiram a mesma metodologia descrita para o solo.

O tamanho de cada parcela experimental foi de $20 \mathrm{~m}$ lineares, tendo cada tratamento 4 repetições, totalizando 80 $\mathrm{m}$ lineares por tratamento. $\mathrm{O}$ experimento obedeceu ao delineamento em blocos casualizados.

Por ocasião da colheita (04/11/2002) contabilizou-se o total de frutos, sendo amostrados 4 frutos por repetição, totalizando assim 16 frutos por tratamento. Estes foram devidamente etiquetados e enviados para análise póscolheita no laboratório da ESAM. As variáveis analisadas foram: peso dos frutos (PF), peso estimado (PE), diâmetro longitudinal (DL), diâmetro transversal (DT), espessura de polpa (EP), sólidos solúveis totais (SST) e firmeza de polpa (FP). O conteúdo de SST foi determinado por leitura em refratômetro ótico, modelo ATAGO N - 1E (0-32\%), registrado com precisão de 0,1 a $25^{\circ} \mathrm{C}$, expressando os resultados em porcentagem (\%). A firmeza da polpa foi medida como resistência à penetração usando-se penetrômetro McCormick mode- 
Tabela 1. Valores médios das variáveis: peso do fruto (PF), peso estimado da produção (PE), diâmetro longitudinal (DL), espessura de polpa (EP) e sólidos solúveis (SST) para o melão de melão AF 646 cultivado sob condições de campo. Mossoró, ESAM, 2002.

\begin{tabular}{cccccc}
\hline \multirow{2}{*}{ Trat $^{2}}$. & \multicolumn{5}{c}{ Características } \\
\cline { 2 - 6 } & $\mathbf{P F}(\mathbf{k g})$ & PE $^{3}\left(\mathbf{t} . \mathbf{h a}^{-1}\right)$ & $\mathbf{D L}(\mathbf{c m})$ & $\mathbf{E P}(\mathbf{c m})$ & SST $(\%)$ \\
\hline 1 & $2,03 \mathrm{a}^{1}$ & $46,06 \mathrm{a}$ & $17,1 \mathrm{a}$ & $4,0 \mathrm{a}$ & $9,3 \mathrm{a}$ \\
2 & $1,94 \mathrm{a}$ & $45,71 \mathrm{a}$ & $17,2 \mathrm{a}$ & $4,1 \mathrm{a}$ & $9,3 \mathrm{a}$ \\
3 & $1,83 \mathrm{a}$ & $42,20 \mathrm{a}$ & $16,5 \mathrm{a}$ & $4,0 \mathrm{a}$ & $10,0 \mathrm{a}$ \\
4 & $1,84 \mathrm{a}$ & $37,03 \mathrm{a}$ & $16,8 \mathrm{a}$ & $4,0 \mathrm{a}$ & $8,8 \mathrm{a}$ \\
5 & $1,85 \mathrm{a}$ & $42,43 \mathrm{a}$ & $16,6 \mathrm{a}$ & $3,9 \mathrm{a}$ & $9,4 \mathrm{a}$ \\
6 & $1,26 \mathrm{~b}$ & $25,83 \mathrm{~b}$ & $14,9 \mathrm{~b}$ & $3,3 \mathrm{~b}$ & $6,9 \mathrm{~b}$ \\
\hline
\end{tabular}

${ }^{1}$ Médias seguidas da mesma letra, na vertical, não diferem entre si pelo teste Tukey ao nível de $5 \%$ de probabilidade; ${ }^{2} 1=500$ kg.ha ${ }^{-1}$ de $\operatorname{Ribumin}^{\circledast}(\mathrm{Rb}) ; 2=750 \mathrm{~kg}^{-h a^{-1}}$ de Rb; $3=1.000$ kg.ha ${ }^{-1}$ de Rb; $4=7.000$ kg.ha ${ }^{-1}$ de Pole $^{\circledast}(\mathrm{P}) ; 5=500$ kg.ha ${ }^{-1}$ de Rb +3.500 kg.ha ${ }^{-1}$ de P; $6=$ testemunha absoluta. ${ }^{3} \mathrm{O}$ valor da produção estimada foi calculado pela fórmula $\mathrm{PE}=(\mathrm{NF}$ ha $1 * \mathrm{PF})$; sendo $\mathrm{NF}$ ha $^{-1}=\mathrm{PE} / \mathrm{PF}$.

Tabela 2. Análise econômica dos tratamentos (fertilizantes) aplicados. Mossoró, ESAM, 2002.

\begin{tabular}{ccc}
\hline Tratamentos $^{1}$ & Custo $^{1}$ Total (CT) $\left(\mathbf{R} \$\right.$.ha $\left.^{-1}\right)$ & Produtividade (t.ha $\left.^{-1}\right)$ \\
\hline 1 & 157,50 & 46,06 \\
3 & 315,00 & 45,71 \\
4 & 472,50 & 42,20 \\
5 & 560,00 & 37,03 \\
6 & 437,50 & 42,43 \\
& 0,0 & 25,83 \\
\hline
\end{tabular}

${ }^{1}(1) 500$ kg.ha-1 de Ribumin ${ }^{\circledR}$ (Rb); (2) 750 kg.ha' de Rb; (3) 1.000 kg.ha' ${ }^{-1}$ de Rb; (4) 7.000 kg.ha ${ }^{-1}$ de Pole ${ }^{\circledR}$ (P); (5) 500 kg.ha-1 de Rb +3.500 kg.ha ${ }^{-1}$ de P; (6) testemunha absoluta.

lo FT 327 com plunger de ponta cônica de $8 \mathrm{~mm}$ de diâmetro (valor máximo de leitura 27 libras) tomando-se duas leituras eqüidistantes em cada uma das metades equatoriais do fruto. Os resultados foram obtidos em libras e convertidos para Newton $(\mathrm{N})$ utilizando-se o fator de conversão 4,45 (GOMES JÚNIOR et al., 2001).

Também foram estimados os valores de produtividade ( $\mathrm{NF} \mathrm{ha-1)}$ baseado no número médio de frutos produzidos (NF) e o peso médio estimado na parcela experimental (PE), pela fórmula $\mathrm{PE}=\left(\mathrm{NF} \mathrm{ha}^{-1} \mathrm{PF}\right)$. Estes valores contribuíram para uma melhor visualização final do ensaio, onde foram avaliados os custos de produção dos tratamentos (fertilizantes orgânicos) utilizados.

Os dados obtidos foram submetidos à análise de variância e as médias comparadas pelo teste de Tukey ao nível de $5 \%$ de probabilidade.

\section{RESULTADOS E DISCUSSÃO}

A análise de variância detectou diferença significativa, ao nível de $5 \%$ de probabilidade, para as variáveis $\mathrm{PF}, \mathrm{PE}$, DL, EP e SST. Entretanto, em relação ao número de frutos (NF), DT e FP os dados não apresentaram diferenças significativas, com intervalo médio de 82,0 a 90,75 frutos por parcela (NF). Estimou-se uma produtividade que variou de 20,13 a 23,56 t.ha-1 (NF ha $\left.{ }^{-1}\right)$. Os valores de DT e FP apresentaram valores compreendidos entre 12,8 a $15,2 \mathrm{~cm} \mathrm{e}$ 31,5 a 32,97 N, respectivamente.

Entre as variáveis quantitativas, as médias do DL e da EP apresentaram diferença significativa entre a testemunha e os demais tratamentos, que não apresentaram diferença entre si (Tabela 1), indicando que a menor dose do fertilizante Ribumin ${ }^{\circledR}$ já apresentou resposta significativa.

A variável FP não apresentou diferença estatística significativa entre as médias dos tratamentos, sendo observada uma variação de 32,97 a 32,21 N, mas o valor mínimo $(24 \mathrm{~N})$ recomendado para frutos destinados à exportação (FILGUEIRAS et al., 2001) foi alcançado, em todos os tratamentos, inclusive na testemunha.
A produtividade foi considerada alta em relação à média nacional $\left(18,20 \mathrm{t} \mathrm{ha}^{-1}\right)$ e do Nordeste brasileiro $\left(20,60 \mathrm{t} \mathrm{ha}^{-1}\right)$ segundo IBGE (2000), sendo maior na presença de fertilizante orgânico independente da fonte utilizada. A produtividade do tratamento 1 (menor dose do Ribumin $^{\circledR}$ ) foi 1,8 vezes superior à produtividade da testemunha. No entanto, não houve diferença entre as médias dos tratamentos para número de frutos.

O solo da área onde o experimento foi instalado é extremamente arenoso com baixos teores de $\mathrm{Ca}, \mathrm{Mg}$ e $\mathrm{K}$, baixas CTC e capacidade de retenção de água. Nesse caso, a adição de matéria orgânica, segundo Moreira e Siqueira (2002), é crucial para o aumento da produtividade e constitui o alicerce da sustentabilidade agrícola. Ela atua como agente cimentante formando e estabilizando os agregados do solo, melhorando sua estrutura e, conseqüientemente aumentando sua capacidade de retenção de água. Além disso, aumenta a CTC do solo permitindo a permanência dos nutrientes no sistema, podendo também reduzir o $\mathrm{pH}$ do solo disponibilizando alguns micronutrientes.

Quanto ao peso médio dos frutos, observa-se que a testemunha diferiu dos demais tratamentos onde se aplicou fertilizante orgânico, apresentando menor peso médio. Os valores variaram de 1,26 a 2,03 kg (Tabela 1), e segundo Gorgatti Neto et al. (1994) são recomendados frutos com até $2,00 \mathrm{~kg}$ de peso, sendo esta característica determinante para a comercialização do produto, pois, atualmente, a maior demanda de frutos de melão pelo mercado interno é do tipo 6 a 8 e do mercado externo de 8 a 10 .

Detectou-se diferença significativa entre os teores médios de SST da testemunha e os demais tratamentos que não diferiram entre si (Tabela 1). Os teores médios apresentados pela cultivar em estudo oscilaram entre $6,9 \%$ e $10,0 \%$, sendo maiores nos tratamentos onde se aplicou fertilizante orgânico. A exigência do teor de SST para o mercado externo é de no mínimo 9,0\% (FILGUEIRAS et al., 2001), sendo considerados não comercializáveis os frutos com teores abaixo desse valor (GORGATTI NETO et al., 1994).

Apesar de não ter sido constatada diferença estatística significativa entre 
as médias de produtividade, para os tratamentos onde se adicionou fertilizante orgânico, observa-se, na Tabela 2, que o fertilizante orgânico Ribumin ${ }^{\circledR}$ proporcionou baixos custos de produção. Quando se utilizou a menor dose (500 kg/ha) desse fertilizante, o custo de produção foi de $\mathrm{R} \$$ 3,42 por cada tonelada de fruto produzido, sendo o menor quando comparado com os demais tratamentos. $\mathrm{O}$ maior custo de produção foi alcançado no tratamento onde se utilizou o fertilizante Pole ${ }^{\circledR}(\mathrm{R} \$$ 15,12) como única fonte orgânica, sendo aproximadamente 4,4 vezes maior que o do tratamento citado anteriormente. No entanto os custos foram reduzidos, para cerca de $\mathrm{R} \$ 10,31$ pela utilização conjunta dos dois fertilizantes. Diante desse fato e da não detecção de diferenças na qualidade dos frutos, no que diz respeito à firmeza de polpa e teor de sólidos solúveis totais, pode-se considerar o tratamento 1 (Ribumin ${ }^{\circledR} 500 \mathrm{Kg} / \mathrm{ha}$ ) como sendo o melhor tratamento por proporcionar um sistema produtivo que apresente redução de custos, aumento de produtividade, alcançando os padrões mínimos de qualidade dos frutos exigidos no mercado internacional.

\section{LITERATURA CITADA}

EMBRAPA. Manual de métodos de análise de solo. 2. ed. Rio de Janeiro: Centro Nacional de Pesquisa de Solos, 1997. 212 p.

FABEIRO, C.; OLALLA, F.M.S.; JUAN, J.A

Production of muskmelon (Cucumis melo L.) under controlled deficit irrigation in a semi-aric climate. Agricultural water management. Fresno, v.54, p.93-105, 2002.

FILGUEIRAS, H.A.C.; MENEZES, J.B.; ALVES, R.E.; COSTA, F.V.; PEREIRA, L.S.E.; GOMES JÚNIOR, J. Colheita e manuseio póscolheita. In: Alves, R.E. (Org.) Melão. Pós-colheita. Embrapa Agroindústria Tropical (Fortaleza CE). - Brasília: Embrapa Comunicação para Transferência de Tecnologia, 2000. p.23-41; (Frutas do Brasil, 10).

GOMES JÚNIOR, J.; MENEZES, J.B.; NUNES G.H.S.; COSTA, F.B.; SOUZA, P.A. Qualidade pós-colheita do melão tipo Cantaloupe, colhido em dois estádios de maturação. Horticultura Brasileira, Brasília, v.19, n.3, p.356-360, 2001.

GORGATTI NETO, A.; GAYET J.P.; BEINROTN, E.W.; MATALLO, M.; GARCIA E.E.C.; GARCIA, A.E.; ARDITO, G.F.G. BORDIN, M.R. Melão para exportação: procedimento de colheita e pós-colheita. Brasília: Embrapa-SPI/Frupex, 1994. 37 p (Frupex,Publicações Técnicas, 6).

IBGE. Produção agrícola. Disponível em: <http:/ /sidra.ibge.gov.br/cgi-bim/v/m/pamltap >. Acesso em:12 set. 2000 .
MENEZES, J.B.; CASTRO, E.B.; PRAÇA. E.F.; GRANGEIRO, L.C.; COSTA, L.B.A. Efeito do tempo de insolação pós-colheita sobre a qualidade do melão amarelo. Horticultura Brasileira, Brasília, v.16, n.1, p.80-81, 1998.

MENEZES, J.B.; FILGUEIRAS, H.A.C.; ALVES, R.E.; MAIA, C.E.; ANDRADE, G.G.; ALMEIDA, J.H.S.; VIANA, F.M.P. Características do melão para exportação. In: ALVES, R.E. (Org.) Melão. Pós-colheita. Embrapa Agroindústria Tropical (Fortaleza, CE). Brasília: Embrapa Comunicação para Transferência de Tecnologia, 2000. p.10-22; (Frutas do Brasil, 10).

MIRANDA, F.R.; BLEICHER, E. Evapotranspiração e coeficientes de cultivo e de irrigação para a cultura do melão (Cucumis melo L.) na Região Litorânea do Ceará. Fortaleza: Embrapa Agroindústria Tropical, 2001. 17 p. (Boletim de pesquisa e desenvolvimento/Embrapa Agroindústria Tropical, n. 2).

MOREIRA, F.M.S.; SIQUEIRA, J.O. Microbiologia e Bioquímica do solo. Lavras: Editora UFLA, 2002. $626 \mathrm{p}$

PROTRADE. Melons - Export Manual: Tropical fruits and vegetables. Eschborn: GTZ, 1995, 36 p. RICHARDS, L.A. Methods of measuring soil moisture tension. Soil Science, Baltimore: v.68, p.95-112, 1949.

SECEX/DTIC. Secretaria de Comércio Exterior. Disponível em: < http://www.ibraf.org.br/x-es/festa.html>. Acesso em: 20 mai. 2004.

WALKLEY, A.; BLACK, I.A. An examination of the Degtjareff method for determining soil organic matter and proposed modification of the chromic acid titration method. Soil Science, Baltimore, v.37, p.29-38, 1934. 